\title{
Necessidades de cuidados de enfermagem do cuidador da pessoa sob cuidados paliativos
}

Nursing care needs of the caregiver of persons under palliative care

Necesidades de cuidados de enfermería del cuidador de la persona bajo cuidados paliativos

João Vicente César Fonseca, Teresa Rebelo'

'Escola Superior de Enfermagem. Lisboa, Portugal

Submissão: 10/08/2009

Aprovação: 10/07/2010

\section{RESUMO}

O objetivo foi identificar as necessidades de cuidados de enfermagem do cuidador da pessoa em fase terminal e respectivas intervenções de enfermagem, recorrendo a uma revisão sistemática da literatura. Foi efectuada uma pesQuisa na EBSCO e ProQuest e procurados artigos científicos em texto integral, publicados entre janeiro de 1998 a dez de 2008, usando as seguintes palavras-chave: "Palliative care", "Family", "Nursing" e "Needs". Foi utilizado o método de PI[C]OD e seleccionados 14 artigos do total de 77 . As necessidades do cuidador da pessoa em fase terminal são: comunicação; relação de confiança e segurança; reconhecimento e operacionalização de desejos; preparação para o luto; necessidades de informação; capacitação; envolvimento nos cuidados; necessidades emocionais; necessidades espirituais e necessidades de descanso. Concluiu-se que a família apresenta diferentes necessidades Que requerem uma intervenção personalizada do enfermeiro, através do estabelecimento de uma relação de confiança.

Descritores: Cuidados Paliativos; Família; Enfermagem.

\section{ABSTRACT}

The purpose was to identify needs for nursing care of caregiver of the person in the terminal stage and nursing interventions, using a systematic review of the literature. A research in EBSCO and ProQuest and sought Full Text scientific articles, published between 1998 and 2008, using the following keywords: "Palliative care", "Family", "Nursing" and "Needs" was made. The method PI[C]OD was used to select 14 items of total 77. The care needs the person in the terminal stage are: communication, relationship of trust and security, recognition and operationalization of desires; preparation for mourning; needs for information, training, involvement in care, emotional needs, spiritual needs and resting needs. It was concluded that the family has different needs that require personal intervention of the nurse, through the establishment of a trust relationship.

Key words: Palliative care; Family; Nursing.

\section{RESUMEN}

El objetivo fue identificar las necesidades de cuidados de enfermería del cuidador de la persona en fase terminal y sus intervenciones de enfermería, mediante una revisión sistemática de la literatura. Se realizó una búsqueda en EBSCO y ProQuest y busco Texto completo de artículos científicos, publicados entre el 1998 y el 2008, mediante el siguientes palabras clave: "Palliative care", "Family", "Nursing” y "Needs". El método PI[C]OD fueutilizado para seleccionar los 14 elementos seleccionados del total de 77 . Las necesidades del cuidador de la persona en fase terminal fueran: la comunicación, la relación de confianza y la seguridad, el reconocimiento y la puesta en práctica de los deseos, la preparación para el duelo; necesidades de información, formación, participación en la atención, necesidades emocionales, espirituales y las necesidades de descanso. Se hay concluydo que la familia tiene diferentes necesidades que requieren la intervención personal de enfermería, mediante el establecimiento de una relación de confianza.

Descriptores: Cuidados paliativos; Familia; Enfermería. 


\section{INTRODUÇÃO}

Na prestação de cuidados à pessoa em fase terminal destacase a importância do enfermeiro acompanhar a família/cuidador ao longo do seu processo de luto, porQue esta vivencia um grande sofrimento emocional, ao mesmo tempo Que, deverá ser capaz de reunir forças para ajudar a pessoa em fase terminal. Assim sendo, a família deverá ser informada sobre a evolução da doença, alertada para possíveis sintomas e para o desenrolar da etapa final ${ }^{(1)}$. Tendo em conta a complexidade das vivências da família/cuidador da pessoa em fase terminal e a pertinência do enfermeiro dar respostas às suas necessidades, formulamos uma Questão de partida, "Quais as necessidades de cuidados de enfermagem do cuidador da pessoa em fase terminal"? Desta forma, temos como objectivo identificar essas necessidades e compreender de Que modo os enfermeiros na sua prática podem dar resposta às mesmas.

A morte é uma realidade complexa: "houve um início para cada um de nós e haverá um fim" (2). A pessoa ao consciencializarse de Que a sua morte está iminente vivencia sentimentos e emoções muito fortes, de perda, medo, ansiedade e incerteza, tendo necessidade de ser ouvida, compreendida e acompanhada ${ }^{(3)}$. Surge para dar resposta a essas necessidades os cuidados paliativos, cujas componentes principais são: o alívio dos sintomas; o apoio psicológico, espiritual e emocional; o apoio à família; o apoio durante o luto e a interdisciplinaridade ${ }^{(4)}$. A unidade alvo dos cuidados paliativos será sempre o binómio pessoa em fase terminal/ família, sendo esta prestadora e receptora de cuidados, Que se deverão prolongar ao longo do processo de luto ${ }^{(4-5)}$. O diagnóstico de uma doença grave e a certeza da morte no seio de uma família Quebra a sua homeostasia, o Que tem repercussões no seu funcionamento e estrutura, gerando tensão e exigindo processos de adaptação ${ }^{(3,6-12)}$. Assim, a família necessita de ajuda para se adaptar à situação, ter capacidade para apoiar a pessoa em fase terminal $^{(7)}$, manter com a mesma uma comunicação verdadeira e afectuosa, lidar com as crises Que esta poderá atravessar e, por fim, realizar um luto saudável(3). Deverá então ser envolvida nos cuidados, através do investimento na comunicação e no estabelecimento de uma relação de parceria, interactiva, dinâmica,

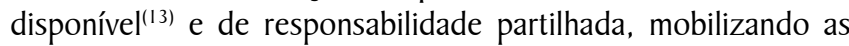
capacidades e potencialidades da mesma como um recurso ${ }^{(7)}$. O cuidador principal "refere-se à pessoa, familiar ou não, Que proporciona a maior parte dos cuidados à pessoa em situação de dependência, sem receber QualQuer compensação econômica"(14). Este, em geral, "acaba por se dividir entre as responsabilidades profissionais, conjugais, sociais e familiares"(15). Cuidar de um familiar em fase terminal é desgastante ${ }^{(16)}$, com custos físicos, psíquicos, sociais e financeiros, contudo, é frequentemente referido pelos seus cuidadores como um tempo único e gratificante, promotor do amadurecimento e crescimento pessoal e espiritual ${ }^{(9,17-18)}$.

\section{MÉTODOS}

Com a finalidade de sistematizarmos o conhecimento existente sobre as necessidades de cuidados de enfermagem do cuidador da pessoa em fase terminal, formulamos uma Questão de partida, "Em relação ao cuidador da pessoa em fase terminal (P), em termos de cuidados de enfermagem (I), Quais as necessidades descritas (O)?" (formato PICO(D)). A partir desta Questão identificamos as seguintes palavras-chave: palliative care, family, needs, nursing, sendo Que, através do cruzamento das mesmas, realizamos uma pesquisa na Ebsco e na Proquest, nos últimos 10. Base de dados electrônica observada: EBSCO (CINAHL Plus with Full Text; MEDLINE with Full Text; British Nursing Index; Nursing \& Allied Health Collection: Comprehensive; Cochrane Database of Systematic Reviews). Foram consultadas tendo em conta o intervalo temporal entre 1998 e 2008, usando as seguintes palavras-chave: Palliative care, Family, Nursing e Needs, Que foram procuradas no resumo. Resultado: 50 artigos. Base de dados electrónica observada: PROQUEST (Nursing \& Allied Health Source). Foram consultadas tendo em conta o intervalo temporal entre 0I/0I/ 1998 e 3 1/12/2008, usando as seguintes palavras-chave: Palliative care, Family, Nursing e Needs, Que foram procuradas na citação e resumo (abstract). Resultado: 27 artigos.

Para obtermos os artigos adequados à Questão de investigação e à finalidade do estudo, estabelecemos os critérios de inclusão dos artigos Que abordassem as seguinte temáticas: Pessoa em fase terminal; Familiares da pessoa em fase terminal; Profissionais de saúde Que contactam e prestam cuidados a pessoas em fase terminal (enfermeiros); Necessidades da família da pessoa em fase terminal; Sentimentos e vivências dos familiares; Cuidados prestados aos familiares; Vivências dos profissionais de saúde na prestação de cuidados paliativos. De um total de 77 artigos, apenas 14 correspondem aos critérios de inclusão, sendo Que, os restantes foram excluídos pelas seguintes razões: 23 não são estudos de investigação; cinco não são texto completo; 31 não se adeQuam aos critérios de inclusão; e Quatro são artigos repetidos. Assim sendo, fazem parte da nossa revisão sistemática da literatura 14 artigos de investigação (Quadro I).

\section{RESULTADOS E DISCUSSÃO}

A informação resultante da análise crítica do conjunto de artigos seleccionados foi reunida em tabelas retiradas da Cochrane Database of Systematic Reviews, por facilitarem a sua sistematização e apresentação de modo objectivo. Estes artigos contribuíram para dar resposta à nossa pergunta de partida, uma vez Que, explicita ou implicitamente, na sua maioria, abordam as necessidades da família/cuidador da pessoa em fase terminal. As três preocupações major a considerar para a melhoria da Qualidade dos cuidados paliativos são: ênfase nos cuidados espirituais, envolvimento da família e educação dos profissionais ao nível da ética ${ }^{(19)}$. No sentido de apoiar e preparar precocemente a pessoa e família para a trajectória final da doença, recomenda-se uma melhor educação da pessoa e família, bem como uma melhor colaboração entre os membros da equipa multidisciplinar ${ }^{(20)}$, sendo importante melhorar a comunicação entre a família e os profissionais de saúde acerca das decisões sobre o tratamento ${ }^{(21)}$.

É de realçar o envolvimento de um ou mais membros da família na prestação de cuidados físicos (higiene, alimentação, eliminação, alternância de decúbitos) e no controlo dos sintomas, ajudando ou substituindo a pessoa em fase terminal na realização de actividades Que antes executava sozinha ${ }^{(22)}$. Neste sentido, destaca-se o papel do cuidador e a importância da necessidade de capacitação do 


\begin{tabular}{|c|c|c|c|}
\hline AUTOR/ANO & SUIEITOS & INTERVENÇÕES & RESULTADOS \\
\hline Weir (1999) & $\begin{array}{l}8 \text { Indivíduos Que cuidaram de } \\
\text { pessoas a receberem cuidados } \\
\text { paliativos por amostragem de } \\
\text { selecção racional. }\end{array}$ & $\begin{array}{l}\text { Abordagem Qualitativa, } \\
\text { estudo descritivo. } \\
\text { Entrevista semi- } \\
\text { estruturada. }\end{array}$ & $\begin{array}{l}\text { Cuidar em casa de um familiar em fase terminal põe em risco a saúde e harmonia do } \\
\text { cuidador pelas responsabilidades Que acarreta. Destaca-se a importância da } \\
\text { compreensão e acompanhamento por parte dos mesmos enfermeiros, bem como a } \\
\text { promoção de momentos de descanso e a elaboração de planos de contingência para } \\
\text { situações de crise inesperadas. }\end{array}$ \\
\hline Forbes et al (2000) & $\begin{array}{l}28 \text { Familiares de pessoas internadas } \\
\text { em enfermarias com demência. }\end{array}$ & $\begin{array}{l}\text { Abordagem Qualitativa, } \\
\text { estudo descritivo. }\end{array}$ & $\begin{array}{l}5 \text { Temas descrevem o contexto de tomada de decisão dos familiares: efeito } \\
\text { emocional, afrontamento da história de vida, as duas faces da morte (tragédia versus } \\
\text { bênção), valores e metas dos cuidados paliativos, e a irreconhecível trajectória da } \\
\text { morte. Estes revelaram um insatisfatório suporte informativo e emocional por parte de } \\
\text { um profissional de forma continuada. }\end{array}$ \\
\hline Luker et al (2000) & $\begin{array}{l}62 \text { Membros da equipa de } \\
\text { enfermagem comunitária. }\end{array}$ & $\begin{array}{l}\text { Abordagem Qualitativa. } \\
\text { Entrevistas estruturadas. }\end{array}$ & $\begin{array}{l}\text { Na comunidade é essencial o enfermeiro conhecer a família/cuidador, através de uma } \\
\text { atitude de abertura e sensibilidade. }\end{array}$ \\
\hline $\begin{array}{l}\text { Reynolds, Kimberly } \\
(2002)\end{array}$ & $\begin{array}{l}\text { Enfermeiros, auxiliares de } \\
\text { enfermagem e membros das famílias. } \\
\text { Total de } 259 \text { participantes. }\end{array}$ & $\begin{array}{l}\text { Abordagem Quantitativa. } \\
\text { Entrevistas estruturadas. }\end{array}$ & $\begin{array}{l}\text { A morte não é apenas um acontecimento individual é também familiar, há Que incidir } \\
\text { a atenção por parte dos profissionais na família, visto Que esta sofre com a doença do } \\
\text { paciente e permanece sempre junto do mesmo. }\end{array}$ \\
\hline $\begin{array}{l}\text { Hudson, McMurray } \\
\text { (2002) }\end{array}$ & $\begin{array}{l}8 \text { Ex-cuidadores e } 6 \text { actuais } \\
\text { cuidadores em casa de pessoas com } \\
\text { cancro em fase avançada, e } 7 \\
\text { enfermeiros comunitários de } \\
\text { cuidados paliativos. }\end{array}$ & $\begin{array}{l}\text { Abordagem Qualitativa. } \\
\text { Discussão em grupo com } \\
\text { moderador. }\end{array}$ & $\begin{array}{l}\text { Os factores Que ajudam os cuidadores a desempenhar o seu papel são o suporte, a } \\
\text { experiência prévia, a relação com a pessoa Que está a morrer e viver um dia de cada } \\
\text { vez. A orientação e suporte deverão ser providenciados precocemente, dando } \\
\text { resposta às necessidades individuais expressas pelo cuidador, utilizando diferentes } \\
\text { meios, documentando, planeando e avaliando. }\end{array}$ \\
\hline $\begin{array}{l}\text { Manosilapakom } \\
\text { (2003) }\end{array}$ & 538 Enfermeiros & $\begin{array}{l}\text { Abordagem Qualitativa e } \\
\text { Quantitativa. Questionário } \\
\text { e narrativas. }\end{array}$ & $\begin{array}{l}\text { Os } 3 \text { problemas mais importantes em cuidados paliativos identificados pelas } \\
\text { enfermeiras são: inabilidade para conhecer as necessidades espirituais, solidão e } \\
\text { controlo da dor. }\end{array}$ \\
\hline $\begin{array}{l}\text { Borneman, Wagman } \\
\text { (2003) }\end{array}$ & $\begin{array}{l}45 \text { Cuidadores familiares de } \\
\text { pacientes com cancro. }\end{array}$ & $\begin{array}{l}\text { Abordagem Qualitativa e } \\
\text { Quantitativa. Entrevistas } \\
\text { semi-estruturadas. }\end{array}$ & $\begin{array}{l}\text { As necessidades dos cuidadores são múltiplas e complexas, exigem uma avaliação } \\
\text { para o planeamento de intervenções. Os enfermeiros deverão encaminhar os } \\
\text { cuidadores para serviços de apoio, como assistentes sociais, psicólogos, grupos de } \\
\text { apoio, aconselhamento e luto. }\end{array}$ \\
\hline $\begin{array}{l}\text { Dunne, Sullivan } \\
(2005)\end{array}$ & $\begin{array}{l}25 \text { Enfermeiras de Saúde } \\
\text { Comunitária. }\end{array}$ & Abordagem eualitativa. & $\begin{array}{l}\text { Foram identificadas Quatro temáticas cruciais: comunicação entre enfermeiro - } \\
\text { doente; família como elemento fundamental do cuidado; controlo dos sintomas e } \\
\text { emoções. }\end{array}$ \\
\hline $\begin{array}{l}\text { Skilbeck et al } \\
(2005)\end{array}$ & $\begin{array}{l}25 \text { Cuidadores familiares em Que a } \\
\text { sua pessoa foi admitida na } \\
\text { Instituição (hospício). }\end{array}$ & $\begin{array}{l}\text { Abordagem Qualitativa e } \\
\text { Quantitativa. Entrevistas } \\
\text { semi-estruturadas }\end{array}$ & $\begin{array}{l}\text { Verificou-se Que os cuidados prestados às pessoas significativas em fim de vida são } \\
\text { exigentes e trabalhosos. Sendo Que, a maioria dos cuidadores, têm um trabalho } \\
\text { árduo, tanto fisicamente como mentalmente, sobressaindo a necessidade de descanso } \\
\text { dos mesmos. }\end{array}$ \\
\hline Street et al (2005) & $\begin{array}{l}\text { Profissionais de saúde e outros } \\
\text { profissionais envolvidos na prestação } \\
\text { de cuidados paliativos. }\end{array}$ & $\begin{array}{l}\text { Abordagem Qualitativa e } \\
\text { Quantitativa. Entrevistas } \\
\text { semi-estruturadas e } \\
\text { Palliative Care Practices } \\
\text { Questionnaire. }\end{array}$ & $\begin{array}{l}\text { As estratégias para o suporte da família incluem: promoção de uma comunicação } \\
\text { efectiva, envolvimento nos cuidados, negociação das necessidades especiais, } \\
\text { providenciar um óptimo controlo da dor e outros sintomas, providenciar suporte } \\
\text { existencial, preparação para a morte, permitir Que participe na morte e providenciar } \\
\text { suporte para o luto. }\end{array}$ \\
\hline $\begin{array}{l}\text { Wilkes, White } \\
(2005)\end{array}$ & $\begin{array}{l}19 \text { Membros de } 17 \text { famílias de } \\
\text { pessoas com cancro e } 10 \\
\text { enfermeiras especialistas em } \\
\text { cuidados paliativos Que trabalham na } \\
\text { mesma área geográfica Que os } \\
\text { anteriores. }\end{array}$ & $\begin{array}{l}\text { Estudo Qualitativo cross } \\
\text { sectional. } \\
\text { Entrevista semi- } \\
\text { estruturada. }\end{array}$ & $\begin{array}{l}\text { O cuidado físico implica Que a família ajude ou substitua a pessoa doente na } \\
\text { realização de actividades eue antes executava sozinha, tais como os cuidados de } \\
\text { higiene, de eliminação, alternância de posição e alimentação. Os enfermeiros } \\
\text { desempenham um papel fulcral na educação e capacitação da mesma, especialmente } \\
\text { no uso das ajudas técnicas e no controlo dos sintomas. }\end{array}$ \\
\hline Wotton et al (2005) & $\begin{array}{l}17 \text { Enfermeiros seniores com } \\
\text { experiência em cuidados paliativos e } \\
\text { cardíacos de } 3 \text { centros de cuidados } \\
\text { agudos e } 5 \text { centros comunitários. }\end{array}$ & $\begin{array}{l}\text { Estudo descritivo - } \\
\text { exploratório. } \\
\text { Entrevista semi- } \\
\text { estruturada. }\end{array}$ & $\begin{array}{l}\text { Destaca-se a necessidade de uma melhor educação do paciente e família, e } \\
\text { colaboração entre os membros da equipa multidisciplinar, de modo a providenciar um } \\
\text { apoio e preparação precoces dos mesmos para a trajectória final da doença. }\end{array}$ \\
\hline Ferrel (2006) & $\begin{array}{l}108 \text { Enfermeiros Que participaram } \\
\text { em } 2 \text { cursos sobre fim de vida. }\end{array}$ & $\begin{array}{l}\text { Abordagem Qualitativa. } \\
\text { Inquérito escrito. }\end{array}$ & $\begin{array}{l}\text { Emerge a necessidade de uma colaboração com o capelão para avaliar e conhecer as } \\
\text { necessidades espirituais do paciente, família e enfermeiros. }\end{array}$ \\
\hline Weigel et al (2007) & $\begin{array}{l}15 \text { I Enfermeiros representando } 7 \\
\text { unidades hospitalares. }\end{array}$ & $\begin{array}{l}\text { Abordagem Quantitativa. } \\
\text { Professional End-of-life } \\
\text { Care Attitude Scale }\end{array}$ & $\begin{array}{l}\text { Os enfermeiros demonstram apreensão em cuidar da pessoa em fase terminal e sua } \\
\text { família, pelo Que, há necessidade de aumentar o treino de competências nesse } \\
\text { âmbito. }\end{array}$ \\
\hline
\end{tabular}

\section{Quadro 1. Estudos Que constituíram a amostra.}

mesmo, tendo emergido os seguintes factores Que ajudam os cuidadores a desempenhar o seu papel: suporte fornecido pela equipa de cuidados paliativos, experiência prévia, Qualidade da relação com a pessoa em fase terminal e viver um dia de cada vez ${ }^{(23)}$. Por sua vez, os obstáculos à preparação para ser cuidador englobam a necessidade súbita de desempenhar esse papel, a responsabilidade associada ao mesmo, as dificuldades de comunicação com os médicos, a sua não inclusão na discussão sobre o plano de tratamento, a natureza imprevisível desse papel, o impacto físico e psicossocial do mesmo, e a frustração de estar à espera da morte ${ }^{(23)}$.

Considera-se como tópicos fundamentais da informação a fornecer aos cuidadores: direitos e opções, demandas, lidar com o stress e implicações emocionais, cuidados com a própria saúde, promover encorajamento/optimismo, Quem contactar para aconselhamento e ajuda, obter suporte da família e amigos, arranjar alguém com Quem possam partilhar a sua experiência, controlar as implicações financeiras, descansar regularmente, acesso a profissionais competentes, administração de terapêutica, manter a pessoa confortável, aspectos práticos do papel de cuidador (uso de equipamentos, cuidados de higiene e alimentação), preparar uma morte em casa, discutir a morte e o morrer, o Que esperar durante a fase de morte, preparação para o luto e aspectos potencialmente positivos de ser cuidador ${ }^{(23)}$. Cuidar de um familiar em fase terminal é uma experiência difícil ao nível físico e mental, pondo em risco a saúde e harmonia do cuidador, pelo que, os momentos de descanso são considerados importantes no sentido de providenciar uma Quebra em relação às responsabilidades como cuidador $\mathrm{e}$ proporcionar um regresso à vida normal ${ }^{(24-25)}$. 
Cuidar de uma pessoa em fase terminal é pois uma experiência muito significativa, englobando as dimensões, psicológica, emocional e espiritual. A incerteza, os medos em relação ao futuro e a perda são as preocupações psicológicas mais comuns do cuidador da pessoa em fase terminal(26). Por outro lado, os familiares desta evidenciam necessidades emocionais não satisfeitas e não estão preparados para tomar decisões relativas aos tratamentos terminais, devido a um insatisfatório suporte informativo e emocional por parte de um profissional de forma continuada ${ }^{(27)}$. São ainda enfatizadas as necessidades espirituais dos mesmos, nomeadamente a colaboração com o capelão para as avaliar e conhecer, assim como, responder às necessidades espirituais dos enfermeiros ${ }^{(28)}$. Destacam-se as seguintes estratégias para o suporte da família: promoção de uma comunicação efectiva, envolvimento da família nos cuidados, negociação das necessidades especiais, providenciar um óptimo controlo da dor e outros sintomas, providenciar suporte existencial, preparar a família para a morte, permitir Que a família participe na morte e providenciar suporte para o luto ${ }^{(29)}$.

Para Que o enfermeiro possa efectivamente pôr em prática as estratégias anteriores, importa pois Que não sejam descuradas as suas vivências emocionais aQuando da prestação de cuidados à pessoa em fase terminal e família, realçando-se ainda a importância do mesmo como elo chave dentro da equipa de cuidados paliativos ${ }^{(30)}$. Os enfermeiros revelam-se apreensivos ao cuidar de pessoas em fase terminal e suas famílias, sendo Que, os níveis de apreensão variam de acordo com o grau profissional, o género, o departamento e a experiência pessoal|(31).

Para a prestação de cuidados paliativos de Qualidade é necessário Que o enfermeiro conheça a pessoa em fase terminal e família, estabelecendo contacto precocemente, garantindo continuidade, investindo no tempo para estar com os mesmos e não se restringindo aos aspectos físicos dos cuidados ${ }^{(32)}$.

\section{CONCLUSÃO E IMPLICAÇÃO NA PRÁTICA PROFISSIONAL}

Com a orientação da pergunta "Em relação ao cuidador da pessoa em fase terminal (P), em termos de cuidados de enfermagem (I), Quais as necessidades descritas $(\mathrm{O})$ ?", realizamos uma análise crítica de todos dos artigos seleccionados e foi-nos possível elaborar uma checklist das necessidades do cuidador da pessoa em fase terminal (Quadro 2), sendo a maioria destas corroboradas pelos autores Que consultamos para a elaboração da fundamentação desta revisão sistemática ${ }^{(3-4,6,9,13,17-18)}$, dando assim resposta a esta pergunta.

Sendo o envolvimento da família/cuidador um dos aspectos essenciais na prestação de cuidados à pessoa em fase terminal, o cuidador deverá ser capacitado para a prestação dos mesmos, algo Que não se tem verificado, visto Que os estudos analisados referem um défice nesta área(19-20,22-23,27,29).

As intervenções de enfermagem à família/cuidador da pessoa em fase terminal estão descritas no Quadro 3, visando ajudar a família/cuidador a lidar com a situação e melhorar a sua Qualidade de vida ${ }^{(26)}$.

Com a elaboração desta revisão sistemática, foi-nos possível nomear as diferentes necessidades do cuidador da pessoa em fase terminal, bem como, identificar as principais intervenções de enfermagem para lhes dar resposta ${ }^{(33-34)}$. A família/cuidador da pessoa em fase terminal apresenta diferentes necessidades, pelo Que, o enfermeiro nas suas intervenções deverá estar desperto para o reconhecimento e satisfação das mesmas, com base no estabelecimento de uma relação de confiança, no desenvolvimento da Enfermagem enQuanto disciplina e profissão.

- Capacitação para a prestação de cuidados à pessoa em fase terminal:

o $\quad$ Cuidados de higiene, alimentação, eliminação, alternância de decúbitos, uso dos equipamentos, controlo dos sintomas e administração de medicação.

- Comunicação:

o Compreensão e negociação e envolvimento nas tomadas de decisão (segurança na equipa multidisciplinar).

- Estabelecimento de uma relação de confiança:

o Reconhecimento e operacionalização dos seus desejos;

o Preparação para o luto;

- Necessidades:

o Acompanhamento e envolvimento na prestação de cuidados.

o Emocionais;

o Espirituais;

o Descanso;

o Informação

Quadro 2. Necessidades identificadas em relação à família/cuidador da pessoa em fase terminal.

- Capacitação(22).

- Conhecimento da família/ cuidador ${ }^{(32)}$.

- Compreensão e no acompanhamento por parte de um enfermeiro de referência ${ }^{(25)}$.

- Envolvimento nos cuidados ${ }^{(19)}$.

- Suporte emocional ${ }^{(27)}$.

- Informação e educação ${ }^{(20,23)}$

- $\quad$ uidados espirituais ${ }^{(19,28)}$.

- Promoção de momentos de descanso ${ }^{(24)}$

Quadro 3. Intervenções sugeridas pelos estudos. 


\section{REFERÊNCIAS}

1. Astudillo W, Mendinueta C, Larraz M. Cuidados generales de enfermería en los pacientes terminales. In: Astudillo W, Mendinueta C, Astudillo E. Cuidados del enfermo en fase terminal y atención a su familia. 4a ed. Madrid: Eunsa; 2002. p. 50-70.

2. Saraiva D. O enfermeiro e a morte. Nursing 2007; 17(227): 24-30.

3. Pacheco $S$. Cuidar da pessoa em fase terminal: perspectiva ética. 2a ed. Loures: Lusociência; 2004

4 Direcção-Geral da Saúde (Portugal). Divisão de Doenças Genéticas, Crónicas e Geriátricas. Programa Nacional de Cuidados Paliativos. Lisboa: DGS; 2005.

4. Barbosa A. Neto I. Manual de cuidados paliativos. Lisboa: Faculdade de Medicina de Lisboa; 2006.

5. Carvalho M. Morte, cuidados paliativos e a família do doente terminal. Nursing 2007; 17: 227: 36-44.

6. Felicíssimo A, Sequeira P. A família como unidade de intervenção em enfermagem. Sinais Vitais 2007; 31-4.

7. Gomes $\mathrm{C}$. O enfermeiro na ajuda à família com um doente terminal. Sinais Vitais 1995; 3: 29-32.

8. Guarda H. Apoio à família. In: Barbosa A. Neto I. Manual de cuidados paliativos. Lisboa: Faculdade de Medicina de Lisboa: 2006. p. 453-61.

9. Lumini J. Dignidade de morrer no hospital: utopia ou realidade? Nursing 2006; 16: 215: 7-12.

10. Soares H. Pereira S. A vivência da fase terminal de vida de um familiar: o contributo do enfermeiro. Pensar Enferm 2008; I2(I): 4I-7.

11. Sociedade Francesa de Acompanhamento e de Cuidados Paliativos. Desafios da enfermagem em cuidados paliativos. Cuidar: Éticas e Práticas. Loures: Lusociência; 2000.

12. Gonçalves A. O enfermeiro e a família: parceiros de cuidados? Nursing 2001; 12(151): 11-7.

13. Bettencourt A. Cuidar da família Que cuida: o papel do enfermeiro no apoio à família cuidadora após a alta. In: Camarro I. Aprendendo o cuidado de enfermagem. Lisboa: Escola Superior de Enfermagem de Maria Fernanda Resende; 2007. p. 359-81.

14. Monis C. Sobrecarga do cuidador informal. Informar 2005; I I (35): 49-56.

15. Roper N. Logan W. Tierney A. Modelo de enfermagem. $3^{\text {a }}$ ed. Alfragide: Mcgraw-Hill de Portugal; 1995.

16. Apóstolo J. A vivência dos familiares de doentes com doença oncológica em fase terminal de vida numa unidade de cuidados paliativos. Rev Invest Enferm 2004; 10: 29-37.

17. Silva J. Quando a vida chegar ao fim: expectativas do idoso hospitalizado e família. Loures: Lusociência; 2006.

18. Manosilapakorn C. Thai nurses' attitudes, knowledge, ethical dilemmas, and clinical judgment related to end-of-life care in
Thailand [dissertation]. Fairfax: George Mason University; 2003.

19. Wotton K, Borbasi S, Redden M. When all else has failed: nurses' perception of factors influencing palliative care for patients with end-stage heart failure. I Cardiovasc Nurs 2005; 20( I): 18-25.

20. Reynolds K, Henderson M, Schulman A, Hanson L. Needs of the dying in nursing homes. I Palliative Med 2002; 5(6): 895901 .

$2 \mathrm{l}$. Wilkes $\mathrm{L}$, White $\mathrm{K}$. The family and nurse in partnership: providing day-to-day care for rural cancer patients. Australian I Rural Health 2005; 13(2): I 2 I-6.

22. Hudson P, Aranda S, McMurray N. Intervention development for enhanced lay palliative caregiver support - the use of focus groups. Eur I Cancer Care 2002; I I (4): 262-70.

23. Skilbeck J, Payne S, Ingleton M, Nolan M, Carey I, Hanson A. An exploration of family carers' experience of respite services in one specialist palliative care unit. Palliative Med 2005; 19(8): 610-8.

24. Weir V. The experiences of caregivers as care receivers while caring at home for family members who are terminally ill [dissertation]. New York: D’youville College; 1999.

25. Borneman T, Chu D, Wagman L, Ferrell B, Juarez G, McCahill $\mathrm{L}$, et al. Concerns of family caregivers of patients with cancer facing palliative surgery for advanced malignancies. Oncol Nurs Forum 2003; 30(6): 997- 1005.

26. Forbes S. Bern-Klug M. Gessert C. End-of-life decision making for nursing home residents with dementia. I Nurs Scholarship 2000; 32(3): 25 I -8.

27. Ferrell B. Understanding the Moral Distress of Nurses Witnessing Medically Futile Care. Oncol Nurs Forum 2006; 33(5): 922-30.

28. Street A, Love A, Blackford J. Managing family centered palliative care in aged and acute settings. Nurs Health Scien 2005; $7(1): 45-55$

29. Dunne K, Sullivan K, Kernohan G. Palliative care for patients with cancer: district nurses' experiences. I Advan Nurs 2005; 50(4): 372-80

30. Weigel C, Parker G, Fanning L, Reyna K, Gasbarra D. Apprehension among hospital nurses providing end-of-life care. I Hospice Palliative Nurs 2007; 9(2): 86-9I.

31. Luker K, Austin L, Caress A, Hallett C. The importance of 'knowing the patient': community nurses' constructions of Quality in providing palliative care. I Advan Nurs 2000; 3 I (4): 775 82.

32. Oliveira AC, Sa L, Silva MJP O posicionamento do enfermeiro frente à autonomia do paciente terminal. Rev Bras Enferm 2007; 60(3): 286-90

33. Souza LB, Souza LEEM, Souza ÂMA. A ética no cuidado durante o processo de morrer: relato de experiência. Rev Bras Enferm 2005; 58(6): 731-4. 